\title{
Surface Modification with Cross-Linked Random Copolymers: Minimum Effective Thickness
}

\author{
Du Yeol Ryu, $*, \dagger$ Jia-Yu Wang, ${ }^{\ddagger}$ Kristopher A. Lavery, ${ }^{\ddagger}$ Eric Drockenmuller, ${ }^{\perp}$ \\ Sushil K. Satija, ${ }^{\S}$ Craig J. Hawker, ${ }^{\perp}$ and Thomas P. Russell $*$,
}

Department of Chemical Engineering, Yonsei University, 134 Shinchon-Dong, Seodaemun-Gu, Seoul 120-749, Korea; Polymer Science and Engineering Department, University of Massachusetts, Amherst, Massachusetts 01003; Reactor Radiation Division, National Institute of Standards and Technology, Gaithersburg, Maryland 60439; and Materials Research Laboratory, University of California, Santa Barbara, Santa Barbara, California 93106

Received December 22, 2006; Revised Manuscript Received March 30, 2007

\begin{abstract}
Ultrathin films of benzocyclobutene (BCB)-functionalized random copolymer with styrene and methyl methacrylate, $\mathrm{P}(\mathrm{S}-r$-BCB- $r$-MMA), with thicknesses ranging from 0 to $10.5 \mathrm{~nm}$, were thermally crosslinked on Si substrates. The penetration of deuterated PMMA (dPMMA) into the P(S-r-BCB-r-MMA) films and the microdomain orientation of PS- $b$-PMMA diblock copolymers on P(S- $r$-BCB- $r$-MMA) coated substrates were investigated by neutron reflectivity (NR) and scanning force microscopy (SFM), respectively. NR measurements on bilayers of dPMMA on cross-linked $\mathrm{P}(\mathrm{S}-r$-BCB-r-MMA) showed that the neutron scattering length density (SLD) at the substrate was equal to that of $\mathrm{P}(\mathrm{S}-r$-BCB-r-MMA) if the $\mathrm{P}(\mathrm{S}-r$-BCB-r-MMA) film was thicker than $\sim 5.5 \mathrm{~nm}$. With decreasing thickness of the P(S-r-BCB-r-MMA) film, the SLD at the substrate increased, characteristic of an increasing penetration of the dPMMA. When thin films of PS- $b$-PMMA diblock copolymer having cylindrical microdomains are placed on surfaces modified with cross-linked films of $\mathrm{P}(\mathrm{S}-r$-BCB- $r$-MMA) thinner than $\sim 5.5 \mathrm{~nm}$, the cylindrical microdomains orient parallel to the surface, whereas for thicker films, the microdomains orient normal to the surface. Both of these results indicate that interfacial interactions are screened when the $\mathrm{P}(\mathrm{S}-r$-BCB- $r$-MMA) film is thicker than $\sim 5.5 \mathrm{~nm}$.
\end{abstract}

\section{Introduction}

Interfacial interactions are critical in controlling the surface wetting and adhesion of synthetic and biological systems as well as in dictating the microdomain orientation in thin block copolymer films. ${ }^{1-8}$ The directed self-assembly of diblock copolymers is particularly important due to the emergence of block copolymer lithography as a simple route to generate scaffolds and templates for nanostructured materials. ${ }^{9-15}$ Control over the microdomain orientation and lateral order is essential in this application, and a number of strategies have been developed to achieve this goal. To this end, controlled interfacial interactions ${ }^{1,16-18}$ and external fields, like electric fields, ${ }^{19-22}$ solvent evaporation, ${ }^{23-27}$ epitaxy, ${ }^{28-30}$ and surface patterning ${ }^{31-36}$ have been used. Perhaps the simplest of these is the control of interfacial interactions where a microphase-separated diblock copolymer is placed on a surface to which a random copolymer, containing the same constituents as the diblock copolymer, has been anchored or covalently attached. Over a narrow range of copolymer compositions of the random copolymer, the interactions of both blocks with the random copolymer anchored to the substrate are balanced, and the microdomains orient normal to the surface. For all other concentrations of components in the random copolymer, the diblock copolymer microdomains orient parallel to the surface. However, covalently attaching the random copolymer to the surface requires substrate-specific chemistries. Recently, an alternate approach was introduced that is independent of the substrate and does not require substrate-

* To whom correspondence should be addressed. E-mail: dyryu@yonsei.ac.kr (D.Y.R.), russell@mail.pse.edu (T.P.R.).

Yonsei University.

University of Massachusetts.

$\S$ National Institute of Standards and Technology.

${ }^{\perp}$ University of California, Santa Barbara. specific chemistries. Here, in the case of styrene (S) and methyl methacrylate (MMA), a random copolymer of S and MMA was prepared where benzocyclobutene repeat units (BCB) are randomly placed along the chain. The $\mathrm{BCB}$ undergoes thermal cross-linking, the film is held to the substrate by van der Waals interactions, and it is insoluble, even in good solvents like toluene. This generalized approach to surface modification is independent of the nature of the substrate and does not require surface-specific chemistries or self-assemblies. The thickness of the cross-linked random copolymer film must also be optimized. From the viewpoint of pattern transfer of diblock copolymer morphologies, minimizing the thickness of the random copolymer films is important to optimize the fidelity of the pattern transfer. Previous studies have shown that, during spin-coating and subsequent thermal annealing, the diblock copolymer penetrates into the random copolymer film and, as such, will come in contact with the substrate if the cross-linked random copolymer film is too thin. ${ }^{37}$ Here, we report studies focused on the determination of this minimum effective thickness using neutron reflectivity and scanning force microscopy. Two complementary studies, the penetration of deuterated poly(methyl methacrylate) (dPMMA) and the microdomain orientation in PS- $b$-PMMA, were investigated as a function of the thickness of the cross-linked random copolymer film.

Random copolymers of styrene (S) and methyl methacrylate (MMA) with 2 mol \% benzocyclobutene (BCB) units randomly incorporated along the backbone (corresponding to $\sim 7 \mathrm{BCB}$ units per chain) were prepared as described previously. This compositon of $\mathrm{BCB}$ was previously found to be optimal in producing a cross-linked fim on a flat surface. ${ }^{37}$ After spincoating, the $\mathrm{BCB}$ in the $\mathrm{P}(\mathrm{S}-r$-BCB-r-MMA) was thermally cross-linked to give a random copolymer network where the thickness of the film was controlled by the concentration of 
Scheme 1. Experimental Scheme for Neutron Reflectivity with d-PMMA Homopolymer and Surface Morphology with PS- $b$-PMMA Copolymer on the Cross-Linked Film of P(S-r-BCB-r-MMA) Copolymer

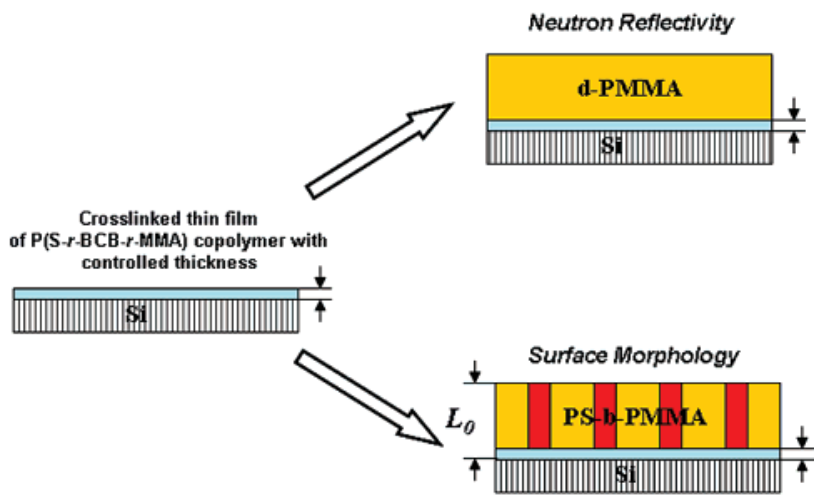

the random copolymer in solution and the spinning speed. Interfacial interactions were tailored by controlling the fraction of S, MMA, and BCB in the random copolymer. For PS- $b$ PMMA diblock copolymers having a 0.28 volume fraction of PMMA, interfacial interactions were balanced with a random copolymer composition of $0.56 / 0.02 / 0.42 .{ }^{37}$ Recently we showed that the contact angles for PS and PMMA were constant for $\mathrm{P}(\mathrm{S}-r$-BCB-r-MMA) thicknesses greater than $\sim 5 \mathrm{~nm}$. The contact angles obtained for PS and PMMA were used to evaluate interfacial energies from Young's equation:

$$
\gamma_{i f}=\gamma_{f}-\gamma_{i} \cos \theta_{i f}
$$

where $\gamma_{i}$ and $\gamma_{f}$ are the surface tensions of the homopolymer $i$ and the $\mathrm{P}(\mathrm{S}-r$-BCB- $r$-MMA) (56/2/42) random copolymer, respectively, and $\theta_{i f}$ is the contact angle of homopolymer $i$ on the cross-linked $\mathrm{P}(\mathrm{S}-r$-BCB- $r$-MMA $)$. It was found that $\gamma_{S f}=0.415 \mathrm{erg} / \mathrm{cm}^{2}$ and $\gamma_{M f}=0.397 \mathrm{erg} / \mathrm{cm}^{2}$. These are almost the same to within experimental error, indicating that the interfacial interactions of PS and PMMA with the P(S-r-BCB$r$-MMA) are balanced. Deviations were found for both PS and PMMA when the $\mathrm{P}(\mathrm{S}-r$-BCB- $r$-MMA) film thicknesses were less than $\sim 5 \mathrm{~nm}$.

Neutron reflectivity was used to assess the penetration of dPMMA into the cross-linked P(S-r-BCB-r-MMA) films with thicknesses from 0 to $10.5 \mathrm{~nm}$. In addition, the orientation of the cylindrical PMMA microdomains in thin films of PS- $b$ PMMA on cross-linked film of P(S-r-BCB-r-MMA) was also measured over a similar range of cross-linked film thicknesses.

\section{Experimental Section}

$\mathrm{P}(\mathrm{S}-r$-BCB- $r$-MMA), synthesized by nitroxide-mediated, living free radical polymerization, ${ }^{38}$ had a number-average molecular weight $\left(M_{\mathrm{n}}\right)$ of 35000 and a polydispersity index (PDI) of 1.18. On average, there were 7 BCB units per chain. The PS- $b$-PMMA, prepared by anionic polymerization, had a $M_{\mathrm{n}}$ of 88000 , PDI of 1.03 , and 0.72 volume fraction of PS. The bulk morphology of the PS- $b$-PMMA consisted of hexagonally packed cylindrical microdomains of PMMA in a PS matrix with a lattice spacing $L_{0}=34.1$ nm. d-PMMA was purchased from Polymer Laboratories Co. $\left(M_{\mathrm{n}}\right.$ $=147000$, PDI $=1.04)$. Solutions of P(S-r-BCB-r-MMA) in toluene were spin-coated onto silicon wafers to produce films with thicknesses $10.5 \mathrm{~nm}$ or less. Subsequently, the P(S-r-BCB-r-MMA) was thermally cross-linked at $250{ }^{\circ} \mathrm{C}$ for 15 min under nitrogen flow to produce robust thin films that were insoluble in toluene. Solutions of d-PMMA and PS- $b$-PMMA were spin-coated onto the cross-linked $\mathrm{P}\left(\mathrm{S}-r\right.$-BCB-r-MMA) and then heated to $170{ }^{\circ} \mathrm{C}$, well above the glass transition temperatures of PS $\left(100^{\circ} \mathrm{C}\right)$ and PMMA $\left(115^{\circ} \mathrm{C}\right)$, for $48 \mathrm{~h}$ under vacuum (Scheme 1).

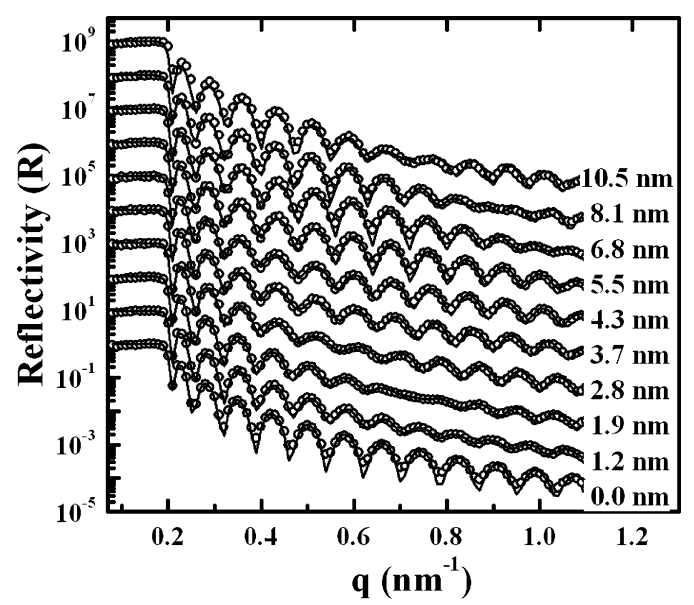

Figure 1. Neutron reflectivity profiles for the bilayers of d-PMMA/ cross-linked film of $\mathrm{P}(\mathrm{S}-r$-BCB- $r$-MMA) copolymer at each different thickness from 0 to $10.5 \mathrm{~nm}$, as indicated.

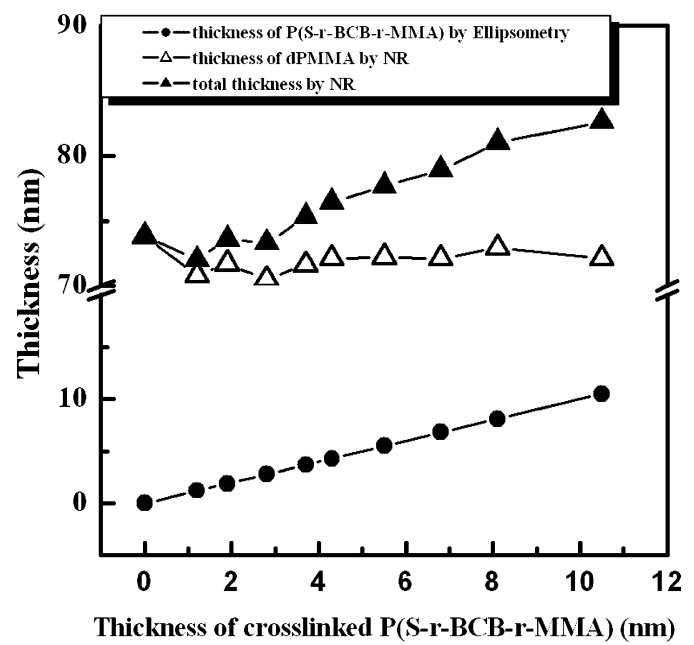

Figure 2. Thickness of the $\mathrm{P}(\mathrm{S}-r$-BCB- $r$-MMA) layer, determined by ellipsometry, and the thickness of the dPMMA layer and total thickness of the bilayer on the surface as a function of the thickeness of the $\mathrm{P}(\mathrm{S}-r$-BCB- $r$-MMA).

The thickness of the P(S-r-BCB-r-MMA) film was measured by ellipsometry (Auto EL II, Rudolph Co.) at a single wavelength of $632.8 \mathrm{~nm}$. Specular neutron reflectivity measurements were performed at the National Institute of Standards and Technology at the National Center for Neutron Research (NCNR) on the NG-7 reflectometer. The wavelength $(\lambda)$ of neutrons was $4.768 \AA$ with $\Delta \lambda / \lambda=0.02$. The interfacial roughness and thickness were obtained by the best fit to the reflectivity profiles. Tapping mode scanning force microscopy (SFM) was performed with a Dimension 3100 (Nanoscope III, Digital Instruments Co.).

\section{Results and Discussion}

Figure 1 shows the NR profiles for bilayers of d-PMMA on cross-linked $\mathrm{P}(\mathrm{S}-r$-BCB- $r$-MMA) films depending on the thickness of the $\mathrm{P}(\mathrm{S}-r$-BCB- $r$-MMA) film. The reflectivity is plotted as a function of the momentum transfer normal to the surface, $q_{z}=4 \pi \sin (\theta / \lambda)$, where $\theta$ is the grazing angle of incidence and $\lambda$ is wavelength. Kiessig fringes, characteristic of the film thickness on the cross-linked film of $\mathrm{P}(\mathrm{S}-r$-BCB-r-MMA) copolymer, are evident, and a clear shift in the interference minima is seen as the thickness of the $\mathrm{P}(\mathrm{S}-r$-BCB- $r$-MMA) film is changed. By fitting the reflectivity profiles, the total thicknesses of the dPMMA and $\mathrm{P}(\mathrm{S}-r$-BCB- $r$-MMA) layers were determined as given in Figure 2. The thickness of the $\mathrm{P}(\mathrm{S}-r$ BCB-r-MMA) layer, determined from ellipsometry, is also 

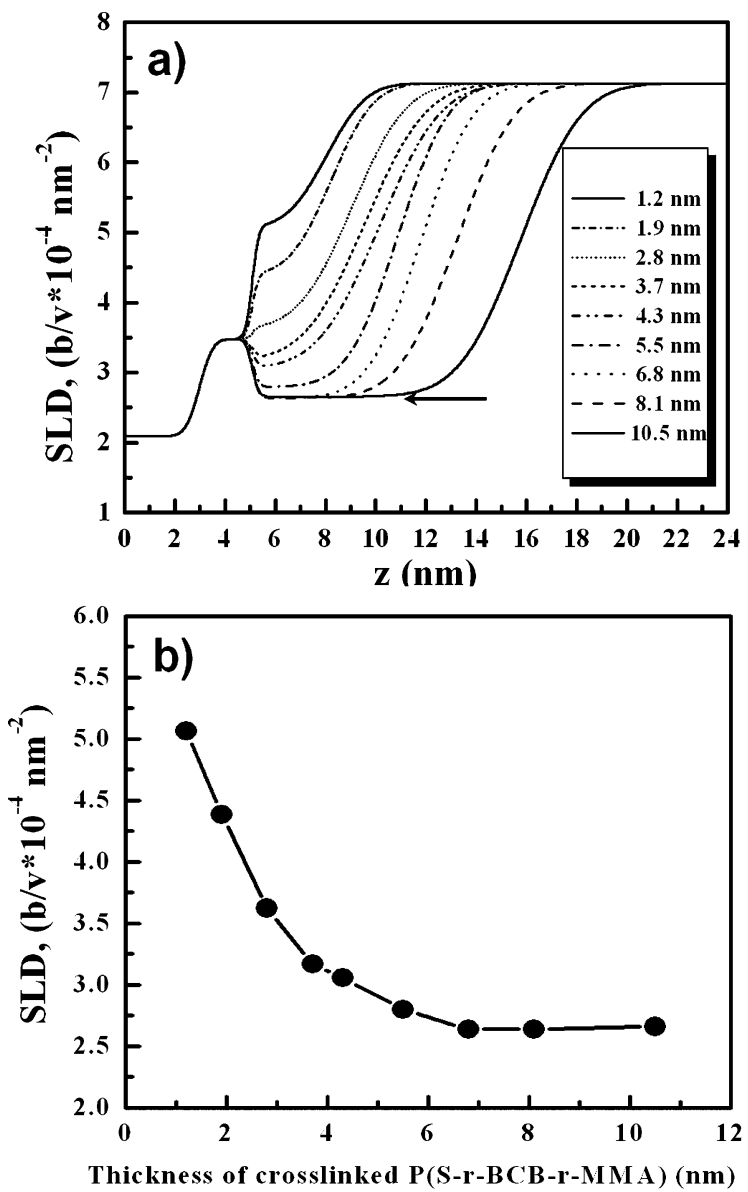

Figure 3. (a) Scattering length density (SLD) profiles obtained from the best fit as a function of distance from the Si substrate, where SLDs of silicon oxide and d-PMMA were set to $3.48 \times 10^{-6}$ and $7.13 \times$ $10^{-6}$, respectively. (b) SLDs for cross-linked film of P(S-r-BCB-rMMA) copolymer depending on initial film thicknesses measured by ellipsometry.

shown. The difference between the two is the thickness of the dPMMA layer, which is constant at a thickness of $72 \pm 1 \mathrm{~nm}$, as would be expected, since the concentration of the dPMMA and preparation conditions remained unchanged.

The scattering length density (SLD) profiles obtained from the best fits to the reflectivity profiles are shown in Figure $3 \mathrm{a}$ as a function of distance $(z)$ from the Si substrate, where the SLDs of Si, native oxide, and d-PMMA were $2.09 \times 10^{-4}, 3.48$ $\times 10^{-4}$, and $7.13 \times 10^{-4} \mathrm{~nm}^{-2}$, respectively. For the $10.5 \mathrm{~nm}$ thick film on cross-linked P(S-r-BCB- $r$-MMA), the SLD of the cross-linked film of $\mathrm{P}(\mathrm{S}-r$-BCB- $r$-MMA) was determined to be $2.65 \times 10^{-4} \mathrm{~nm}^{-2}$ (indicated by the arrow in the figure). When dPMMA was placed on top of the P(S-r-BCB- $r$-MMA), the SLD of the random copolymer was found to attain this value near the substrate, as long as the $\mathrm{P}(\mathrm{S}-r$-BCB- $r$-MMA) film was thicker than $\sim 6 \mathrm{~nm}$. However, the SLD across the P(S-r-BCB$r$-MMA) layer was not constant due to the penetration of the dPMMA into the $\mathrm{P}(\mathrm{S}-r$-BCB-r-MMA). For P(S-r-BCB-r-MMA) film thicknesses of $5.5 \mathrm{~nm}$ or less, the SLD at the substrate increased with decreasing thickness of the P(S-r-BCB-r-MMA), indicating that the IPMMA penetrated through the random copolymer layer to the underlying substrate. The dependence of the SLD at the substrate interface is shown in Figure $3 b$ as a function of the thickness of the cross-linked P(S-r-BCB- $r$ MMA) layer. As can be seen, a significant deviation of the SLD from the SLD of the pure $\mathrm{P}(\mathrm{S}-r$-BCB- $r$-MMA) is seen, once the thickness of the $\mathrm{P}(\mathrm{S}-r$-BCB-r-MMA) layer is less than $\sim 5.5 \mathrm{~nm}$. These data allow us to conclude that a film thickness

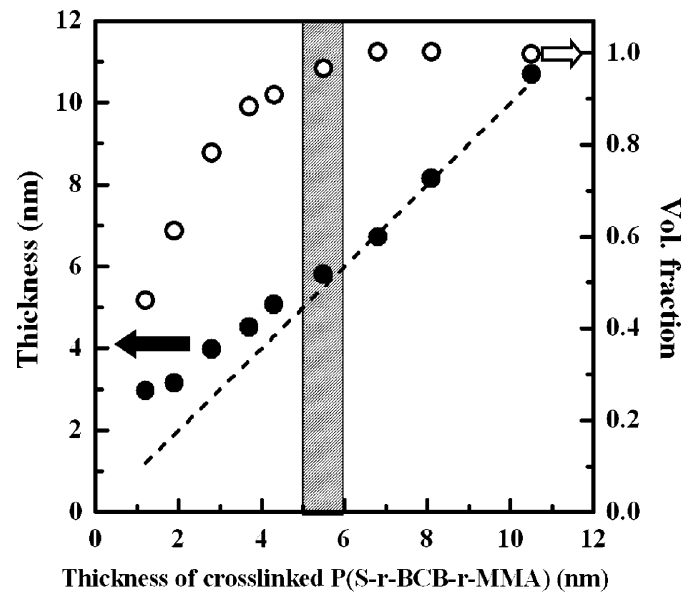

Figure 4. Film thickness (closed symbol) obtained from the best fit to the NR data and volume fraction of the cross-linked $\mathrm{P}(\mathrm{S}-r$-BCB- $r$ MMA) at the substrate as a function of initial thickness of cross-linked film of $\mathrm{P}(\mathrm{S}-r$-BCB- $r$-MMA) copolymer. The dashed line describes linear extrapolation taken from thicker films.

of the cross-linked $\mathrm{P}(\mathrm{S}-r$-BCB-r-MMA) of at least $5.5 \mathrm{~nm}$ is necessary to fully mask the underlying substrate or, in other words, to control interfacial interactions.

The film thickness (closed symbol) obtained from the best fits to the NR profiles is shown in Figure 4 as a function of the film thickness of cross-linked $\mathrm{P}(\mathrm{S}-r$-BCB-r-MMA) along with the film thickness of the cross-linked $\mathrm{P}(\mathrm{S}-r$-BCB-r-MMA), measured by ellipsometry. With decreasing thickness of the cross-linked $\mathrm{P}(\mathrm{S}-r$-BCB- $r$-MMA), the two measurements begin to differ at $\sim 5.5 \mathrm{~nm}$, and the difference increases with decreasing thickness. The volume fraction of dPMMA at the substrate can be calculated from the SLD's of the components, assuming incompressibility, by

$$
\mathrm{SLD}_{\text {mix }}=\operatorname{SLD}_{\mathrm{dPMMA}} \Phi_{\mathrm{dPMMA}}+\operatorname{SLD}_{\text {copolymer }} \Phi_{\text {copolymer }}
$$

where $\operatorname{SLD}_{i}$ and $\Phi_{i}$ are the SLD's and volume fractions of component $i$, respectively. The volume fraction of the crosslinked $\mathrm{P}(\mathrm{S}-r$-BCB-r-MMA) at the substrate obtained from this mixing rule, using SLD values of $2.65 \times 10^{-4}$ and $7.13 \times 10^{-4} \mathrm{~nm}^{-2}$ for P(S-r-BCB-r-MMA) and d-PMMA, respectively, are shown in Figure 4 (open symbols) as a function of the film thickness of cross-linked $\mathrm{P}(\mathrm{S}-r$-BCB- $r$-MMA). For $\mathrm{P}(\mathrm{S}-r$-BCB-r-MMA) thicknesses less than $\sim 5.5 \mathrm{~nm}$, the volume fraction of $\mathrm{P}(\mathrm{S}-r$-BCB- $r$-MMA) decreased with decreasing thickness of cross-linked $\mathrm{P}(\mathrm{S}-r$-BCB-r-MMA). This assumes that the mixing of the dPMMA and $\mathrm{P}(\mathrm{S}-r$-BCB-r-MMA) is uniform laterally. On the basis of these reflectivity results, the shaded region in Figure 4, i.e., for a $\mathrm{P}(\mathrm{S}-r$-BCB- $r$-MMA) thickness of $\sim 5.5 \pm 0.5 \mathrm{~nm}$, is taken as the minimum effective thickness of the cross-linked film necessary to control the substrate interactions and define a surface with a composition, given by the random copolymer. This result has a similar tendency with SLD dependence on the film thickness of crosslinked $\mathrm{P}(\mathrm{S}-r$-BCB- $r$-MMA) shown in Figure $3 \mathrm{~b}$.

Thin films of PS- $b$-PMMA diblock copolymers having cylindrical microdomains of PMMA were spin-coated onto $\mathrm{Si}$ substrates coated with a cross-linked film of $\mathrm{P}(\mathrm{S}-r$-BCB- $r$ MMA) where the thickness of the P(S-r-BCB-r-MMA) was varied. Scanning force microscopy (SFM) studies on the block copolymer films, after thermally annealing the film at $170{ }^{\circ} \mathrm{C}$ for $24 \mathrm{~h}$ under vacuum, are shown in Figure 5. The P(S-r-BCB$r$-MMA) contained a 0.42 mole fraction of MMA segments, which defines the composition where interactions of PS and 

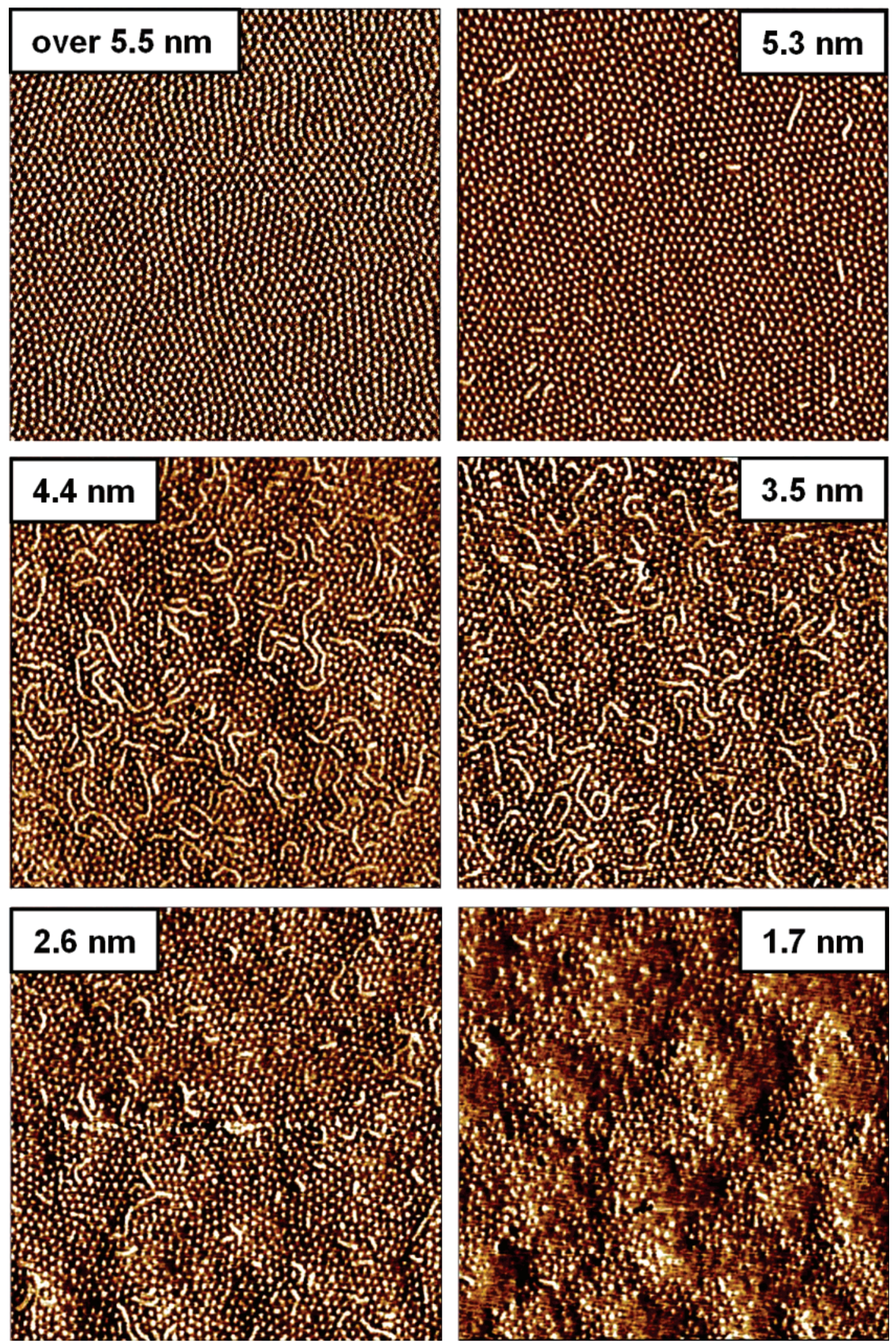

Figure 5. Phase images measured by scanning probe microscopy (SPM) of PS- $b$-PMMA diblock copolymer films on the cross-linked film of $\mathrm{P}(\mathrm{S}-r$-BCB- $r$-MMA) copolymer where the thickness of the cross-linked random copolymer film was varied from 0 to $10.5 \mathrm{~nm}$, as indicated.

PMMA with the substrate are balanced. As can be seen from the SFM images, when the thickness of the cross-linked $\mathrm{P}(\mathrm{S}-r$-BCB-r-MMA) film is $5.5 \mathrm{~nm}$ or greater, the cylindrical microdomains of the PS- $b$-PMMA orient normal to the film surface, indicating that interfacial interactions are balanced.
However, as the thickness of the $\mathrm{P}(\mathrm{S}-r$-BCB- $r$-MMA) film decreases, an increasing number of cylindrical microdomains are seen to orient parallel to the film surface. For P(S-r-BCB$r$-MMA) films less than $\sim 2.6 \mathrm{~nm}$, the ordering and orientation of the cylindrical microdomains degrade. Consequently, for 
$\mathrm{P}(\mathrm{S}-r$-BCB- $r$-MMA) films thicker than $\sim 5.5 \mathrm{~nm}$, the crosslinked film fully shields the underlying substrate, and a surface with balanced interfacial interactions is obtained. For thinner $\mathrm{P}(\mathrm{S}-r$-BCB-r-MMA) films, the diblock copolymer penetrates through the cross-linked random copolymer film and interactions with the underlying silicon oxide substrate cause some of the microdomains to orient parallel to the surface. For very thin $\mathrm{P}(\mathrm{S}-r$-BCB- $r$-MMA) films, the mixing of the PS- $b$-PMMA with the $\mathrm{P}(\mathrm{S}-r$-BCB- $r$-MMA), coupled with interactions with the substrate, leads to a significant degradation of the ordering and orientation of the microdomains. These SFM observations are consistent with the neutron reflectivity results discussed previously.

\section{Conclusions}

Using neutron reflectivity, the penetration of dPMMA into cross-linked films of $\mathrm{P}(\mathrm{S}-r$-BCB- $r$-MMA $)$ was investigated as a function of cross-linked thickness of the $\mathrm{P}(\mathrm{S}-r$-BCB- $r$-MMA) film. For films thicker than $\sim 5.5 \mathrm{~nm}$, the penetration of the homopolymer to the underlying substrate was prevented. By use of SFM, the orientation of the cylindrical microdomains in thin films of an asymmetric PS- $b$-PMMA was also investigated. An orientation of the microdomains normal to the film surface was observed as long as the thickness of the P(S-r-BCB-rMMA) was greater than $\sim 5.5 \mathrm{~nm}$. These combined results indicate that the thickness of the cross-linked $\mathrm{P}(\mathrm{S}-r$-BCB- $r$ MMA) must be greater than $\sim 5.5 \mathrm{~nm}$ to effectively shield interactions with the substrate and, thereby, define interfacial interactions. While results on a silicon/silicon oxide substrate were shown, similar results were also observed on other substrates, like gold, aluminum, and Kapton. Consequently, cross-linked $\mathrm{P}(\mathrm{S}-r$-BCB- $r$-MMA) can be effectively used to manipulate interfacial interactions in a straightforward manner.

Acknowledgment. This work was supported by Ministry of Commerce, Industry and Energy (MOCIE, 10024135-200511), and the Nuclear R\&D Programs funded by the Ministry of Science \& Technology (MOST), Korea, The U.S. Department of Energy, Office of Basic Energy Science, and the National Science Foundation supported Materials Research Science and Engineering Centers at the University of Massachusetts, Amherst, and University of California, Santa Barbara (DMR0520415).

\section{References and Notes}

(1) Mansky, P.; Liu, Y.; Haung, E.; Russell, T. P.; Hawker, C. J. Science 1997, 275, 1458

(2) Wiltzius, P.; Cumming, A. Phys. Rev. Lett. 1991, 66, 3000.

(3) Coulon, G.; Deline, V. R.; Green, P. F.; Russell, T. P. Macromolecules 1989, 22, 2581 .
(4) Anastasiadis, S. H.; Russell, T. P.; Satija, S. K.; Majkrzak, C. F. Phys. Rev. Lett. 1989, 62, 1852.

(5) Genzer, J.; Efimenko, K. Science 2000, 290, 2130.

(6) Chaudhury, M. K.; Whitesides, G. M. Science 1992, 256, 1539.

(7) Chen, C. S.; Mrksich, M.; Huang, S.; Whitesides, G. M.; Ingber, D. E. Science 1997, 276, 1425.

(8) Russell, T. P. Science 2002, 297, 964.

(9) Park, M.; Harrison, C.; Chaikin, P. M.; Register, R. A.; Adamson, D. H. Science 1997, 276, 1401.

(10) Avgeropoulos, A.; Z-H Chan, V.; Lee, V. Y.; Ngo, D.; Miller, R. D.; Hadjichristidis, N.; Thomas, E. L. Chem. Mater. 1998, 10, 2109.

(11) Black, C. T.; Guarini, K. W.; Milkove, K. R.; Baker, S. M.; Russell, T. P.; Tuominen, M. T. Appl. Phys. Lett. 2001, 79, 409.

(12) Mansky, P.; Harrison, C. K.; Chaikin, P. M.; Register, R. A.; Yao, N. Appl. Phys. Lett. 1996, 68, 2586.

(13) Shin, K.; Leach, K. A.; Goldbach, J. T.; Kim, D. H.; Jho, J. Y.; Tuominen, M.; Hawker, C. J.; Russell, T. P. Nano Lett. 2002, 2, 933.

(14) Guarini, K. W.; Black, C. T.; Zhang, Y.; Kim, H.; Sikorski, E. M.; Babich, I. V.; J. Vac. Sci. Technol. B 2002, 20, 2788

(15) Guarini, K. W.; Black, C. T.; Yeung, S. H. I. Adv. Mater. 2002, 14, 1290.

(16) Kim, G.; Libera, M. Macromolecules 1998, 31, 2569.

(17) Kim, G.; Libera, M. Macromolecules 1997, 30, 6810.

(18) Knoll, A.; Magerle, R.; Krausch, G. Macromolecules 2001, 34, 4159.

(19) Lin, Z.; Kim, D. H.; Wu, X-L.; Boosahda, L.; Stone, D.; LaRose, L.; Russell, T. P. Adv. Mater. 2002, 14, 1373.

(20) Mansky, P.; DeRouchey, J.; Russell, T. P. Adv. Mater. 2004, 16, 226.

(21) De Rosa, C.; Park, C.; Thomas, E. L.; Lotz, B. Nature (London) 2000 , $405,433$.

(22) Thurn-Albrecht, T.; DeRouchey, J.; Russell, T. P.; Kolb, R. Macromolecules 2002, 35, 8106.

(23) Kim, G.; Libera, M. Macromolecules 1998, 31, 2569.

(24) Kim, G.; Libera, M. Macromolecules 1998, 31, 2670.

(25) Knoll, A.; Magerle, R.; Krausch, G. Macromolecules 2001, 34, 4159.

(26) Li, Z.; Kim, D. H.; Wu, X-L.; Boosahda, L.; Stone, D.; LaRose, L.; Russell, T. P. Adv. Mater. 2002, 14, 1373.

(27) Kim, S. H.; Misner, M. J.; Kimura, M.; Xu, T.; Russell, T. P. Adv. Mater. 2004, 16, 226.

(28) De Rosa, C.; Park, C.; Thomas, E. L.; Lotz, B. Nature (London) 2000, $405,433$.

(29) De Rosa, C.; Park, C.; Lotz, B.; Wittmann, J. C.; Fetters, L. J.; Thomas, E. L. Macromolecules 2000, 33, 4871.

(30) Park, C.; De Rosa, C.; Fetters, L. J.; Lotz, B.; Thomas, E. L. Adv Mater. 2001, 13, 724.

(31) Segalman, R. A.; Yokoyama, H.; Kramer, E. J. Adv. Mater. 2001, 13, 1152.

(32) Cheng, J. Y.; Ross, C. A.; Thomas, E. L.; Smith, H. I.; Vancso, G. J. Appl. Phys. Lett. 2002, 81, 3657.

(33) Li, Z.; Qu, S.; Rafailovich, M. H.; Sokolov, J.; Tolan, M.; Turner, M. S.; Wang, J.; Schwarz, S. A.; Lorenz, H.; Kotthaus, J. P. Macromolecules 1997, 30, 8410.

(34) Stoykovich, M. P.; Müller, M.; Kim, S. O.; Solak, H. H.; Edwards, E. W.; de Pablo, J. J.; Nealey, P. F. Science 2005, 308, 1442.

(35) Kim, S. O.; Solak, H. H.; Stoykovich, M. P.; Ferrier, N. J.; de Pablo, J. J.; Nealey, P. F. Nature (London) 2003, 424, 411.

(36) Edwards, E. W.; Stoykovich, M. P.; Solak, H. H.; Nealey, P. F Macromolecules 2006, 39, 3598.

(37) Ryu, D. Y.; Shin, K.; Drockenmuller, E.; Hawker, C. J.; Russell, T. P. Science 2005, 308, 236.

(38) Hawker, C. J.; Wooley, K. L. Science 2005, 309, 1200.

MA062939W 